\title{
Assessing the Level of Adoption of a Social Network System for Older Adults
}

\author{
Bilal Ahmad \\ Lero, the Irish Software \\ Research Centre, \\ University of Limerick, Ireland. \\ bilal.ahmad@lero.ie
}

\author{
Ita Richardson \\ Lero, the Irish Software \\ Research Centre, \\ University of Limerick, Ireland. \\ ita.richardson@lero.ie \\ Sarah Beecham \\ Lero, the Irish Software \\ Research Centre, \\ University of Limerick, Ireland. \\ sarah.beecham@lero.ie
}

\author{
Simon McLoughlin \\ IBM Damastown \\ Campus, Dublin, \\ Ireland. \\ simonmcloughlin@ie.ib \\ m.com
}

\begin{abstract}
Existing research indicates that Social Network Systems (SNSs) are a potential way to overcome the social isolation of older adults (OAs). With this in mind, we developed a mobile-based social volunteering network system called GoldenJubilants, taking a user-centred approach. This system connects OAs with volunteer opportunities in the community. The objective of the study presented in this paper is to assess the perceived usability of this newly developed system, focusing particularly on different types of OAs. We employed a mixed method approach with a convenience sample of OAs aged over 55, living in Ireland. The System Usability Scale (SUS) survey was used to test the usability of our system, while semi-structured interviews were conducted to identify suggestions for future improvements. Early results indicate a high level of usability with a suggestion of long-term uptake of this system. Although these preliminary results are based on a small sample, there was a consensus across all participants with a small standard deviation across the results. Thus, our study has proved useful for understanding the level of usability of GoldenJubilants from the lens of OAs. It has also helped to highlight where improvements are required. These findings can guide the designers of SNSs for OAs to create systems that meets their needs, which will in turn have a higher likelihood of being used in practice.
\end{abstract}

Older Adults, system usability scale, usability study, social network systems

\section{INTRODUCTION}

The proportion of OAs is increasing all over the world (United Nations, 2002). This increase in longevity is to be welcomed, but with it comes responsibilities. As a community, we need to ensure this segment of the population remain healthy and engaged (Ageing Well Network, 2012). SNSs such as Facebook, Twitter and WhatsApp have the potential to integrate this segment of population with family, friends and wider community (Burmeister, O.K., 2012; Cornejo et al., 2012; Leist, A.K., 2013), which in turn can improve their health. But, the uptake by OAs is low (Ahmad et al., 2017), due to an array of issues like privacy (Xie et al., 2012), complexity (Lüders \& Brandtzæg, 2017) and lack of purpose (Gibson et al., 2010). Therefore, researchers have proposed special purpose SNSs with the objective of active social engagement such as Fridgenet (Lee et al., 2015) and iDiano (Fang et al., 2015). These are shown to be helpful for OAs during the evaluation period, but the long-term uptake of these systems is unknown. Limiting factors include applications being available in specific languages or having a limited scope (where, for example, diet is the common factor). This situation highlights a clear gap for a system with a broader scope and written in English. Therefore, we proposed and developed a special SNS (Ahmad et al., 2018b), GoldenJubilants based on the requirements elicited earlier (Ahmad et al., 2018a). This system aims to help OAs communicate and encourages active social engagement through suggesting volunteer opportunities to them. The objective of this paper is to present the preliminary results of the usability study we have conducted to understand how easy GoldenJubilants will be for OAs to use. We have chosen the System Usability Scale (SUS) (Brooke, J., 1996) as our measurement tool rather than other available tools (Tullis \& Stetson, 2004; Tullis \& Albert, 2008), because it is quick, reliable with small samples, non-proprietary, technology agnostic and is widely used by practitioners for evaluating usability of mobile and web-based systems (Bangor et al., 2009).

The remainder of the paper is organized as follows. Section 2 describes the research method and Section 3 presents the results. Section 4 discusses the implications of this study along with the threats to validity. Section 5 concludes the study and also discusses future work. 


\section{RESEARCH METHOD}

We employed convenience sampling, recruiting participants through a variety of sources such as press releases and direct contact with retirement villages in Ireland. Figure 1 depicts a total of 20 OAs who participated in our research by age range and gender. Fifty-five percent of them were females and $45 \%$ were males. Eighty-five percent have done some sort of volunteering in the past. Almost half of the participants have completed a third level degree, whereas others have completed a high school or associated degree. Seventy percent live in urban areas and $30 \%$ live in rural areas. Fifty-five percent live with their partners or extended family, whereas $40 \%$ live alone. The other $5 \%$ are living in retirement villages. Seventy percent are fully retired and $30 \%$ are partially retired.

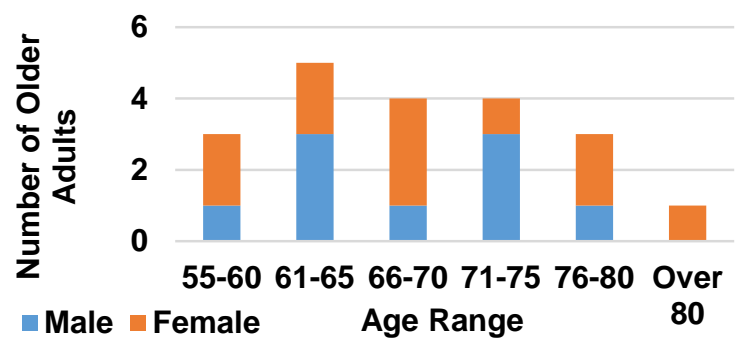

Figure 1: Sample distribution w.r.t. age and gender (\#20)

We conducted usability testing using SUS (Brooke, J., 1996) with these 20 OAs, who tried and tested the GoldenJubilants system. Several sessions were organized between November 2017 to April 2018, to collect data until the point of saturation. The average duration of these sessions was 30 minutes. All of these sessions followed a three step process. Firstly, OAs were given a detailed explanation of GoldenJubilants and instructions about how they can answer SUS. Secondly, the group was split, with some testing a working prototype, and others a paper-based prototype. The reason for doing this was that some OAs have access to and are familiar with technology, while others don't have access to technology yet. Sixteen OAs used the system and performed basic functions such as registration, signing in, looking at volunteer opportunities, adding new opportunities, listening to news and testimonials, and creating a to do list. The other group examined the paper prototype depicting the features that will be available to them. Finally, they all answered the SUS questionnaire (Brooke, J., 1996) along with four semi-structured questions. During this step, OAs asked questions which were answered by the researcher and gave suggestions for potential improvements in the system. SUS, in itself, is a 10-item Likert scale questionnaire with five response options for participants - Strongly disagree to Strongly agree with values 1 to 5 . The positive and negative questions are mixed together, so that the participant has to pay attention before answering. The scoring and analysis of SUS questionnaire is complex. The three steps to analyse and interpret the SUS results are:

(i) Each question is marked from 1 to 5 .

(ii) When the participant answers an odd numbered question, 1 is subtracted from the response. When the participant answers an even question, the answer is subtracted from 5 .

(iii) Results from step 2 are summed and then multiplied by 2.5 , in order to normalize the scores and to achieve percentile ranking. Resulting scores range between 0 to 100 .

We also employed non-parametric statistical techniques to present additional results, as they are suited to our small sample. The SUS questions are listed in the Appendix (Section 7).

\section{RESULTS}

It is recognised that that the SUS questionnaire has good reliability (Lewis \& Sauro, 2009). We used Cronbach alpha $(\alpha)$ to ensure that the SUS questionnaire is measuring the perceived usability of the system effectively. Table 1 presents the overall Cronbach alpha $(0.85)$ for the questionnaire as well as for each individual question. In addition, Figure 2 presents the average SUS results in the form of a radar chart using three facets of measures of central tendency i.e. mean, median and mode. The rationale is to present a convenient summary of the distribution through presenting central values, skewness and outliers. For best positive results, star points are at five, for best negative results, inner vertices are at one. In the case of the mean and the median, the chart is quite similar to a star in comparison with the mode, which is a positive sign. We then calculated the percentile score (described in Section 2) for each question.

Table 1: Cronbach's alpha for SUS questionnaire

\begin{tabular}{|l|l|l|l|l|}
\hline $\begin{array}{l}\text { Overall } \\
\text { Cronbach } \\
\boldsymbol{\alpha}\end{array}$ & $\begin{array}{l}\text { Odd } \\
\text { Questions }\end{array}$ & $\boldsymbol{\alpha}$ & $\begin{array}{l}\text { Even } \\
\text { Questions }\end{array}$ & $\boldsymbol{\alpha}$ \\
\hline 0.8508 & Q1 & 0.84 & Q2 & 0.88 \\
\cline { 2 - 5 } & Q3 & 0.82 & Q4 & 0.83 \\
\cline { 2 - 5 } & Q5 & 0.83 & Q6 & 0.87 \\
\cline { 2 - 5 } & Q7 & 0.83 & Q8 & 0.82 \\
\cline { 2 - 5 } & Q9 & 0.82 & Q10 & 0.81 \\
\hline
\end{tabular}

The mean, median, mode and standard deviation of percentile scores of SUS questionnaire was 74.13, 75,65 and 17.75 respectively. Figure 3 depicts the normal distribution curve, commonly known as bell chart for percentile ranks. We also used a box and whisker plot to present the correlation between gender and percentile rank as shown in Figure 4. 
There is a wide dispersion of score for females in comparison with males. The minimum percentile rank for females is 30 and maximum is 100 . In case of males, the minimum rank is 52.5 and max is 90 .

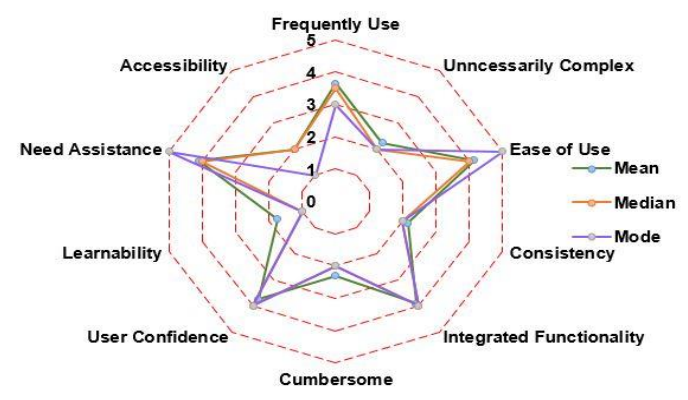

Figure 2: Measures of central tendency for SUS results

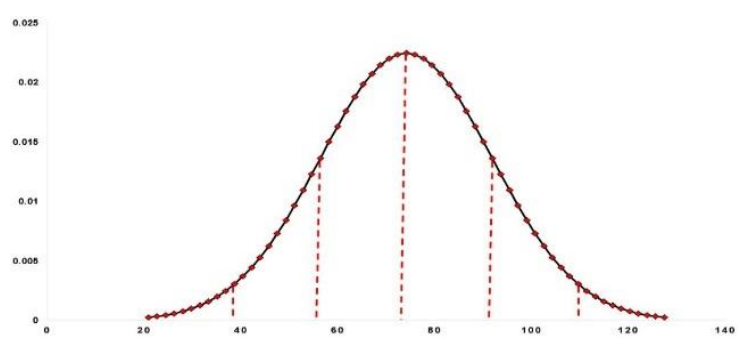

Figure 3: Normal distribution curve for percentile rank

The median for females and males is 82.5 and 65 respectively. Table 2 details the correlation of several demographic parameters on the percentile rank. The median of scores reveal that residence,

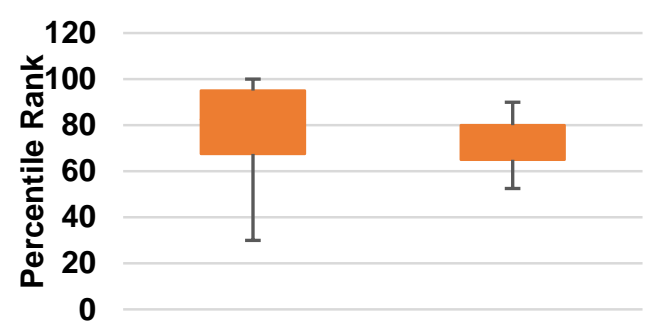

Female Gender Male

Figure 4: Correlation of Gender on percentile score

education, work and health status are directly proportional to the percentile rank. This means that an OA living in an urban area with higher education, working for some hours and is in excellent health condition will have a higher level of perceived usability for GoldenJubilants and vice versa. In depth analysis of the scores reveal a strange finding - that people living alone have a higher perceived usability in comparison with those spending their lives in a retirement village or a care centre. There can be several reasons for that, such as mismatch between the expectations of OAs in these situations. The minimum standard deviation was 2.5 and the maximum was 20.19 for the average percentile ranks. For SUS, a score of at least 70 is considered as passable for a product, whereas over 90 is considered as truly superior (Bangor et al., 2008). If an application has a percentile score of less than 70 , than scrutiny is needed. The median of percentile ranks for all individuals was 75 , which indicate a high level of perceived usability and a conviction by the OAs that system is readily usable.

Table 2: Correlation of demographic parameters with SUS scores (from percentile rank)

\begin{tabular}{|c|c|c|c|c|c|}
\hline \multirow{3}{*}{$\begin{array}{l}\text { Demographic } \\
\text { Residence and } \\
\text { Education Level }\end{array}$} & \multirow[t]{2}{*}{ Sub-parameter } & \multicolumn{4}{|c|}{ Percentile rank } \\
\hline & & \multirow{2}{*}{$\begin{array}{l}\text { Mean } \\
67.91\end{array}$} & \multirow{2}{*}{$\begin{array}{l}\text { Median } \\
65\end{array}$} & \multirow{2}{*}{$\begin{array}{l}\text { Mode } \\
65\end{array}$} & \multirow{2}{*}{$\begin{array}{l}\text { Standard Deviation } \\
15.23\end{array}$} \\
\hline & Rural & & & & \\
\hline & Urban & 76.78 & 82.5 & 65 & 18.08 \\
\hline & High School & 58.33 & 57.5 & 52.5 & 5.13 \\
\hline & Associate Degree & 77.91 & 77.5 & 52.5 & 17.81 \\
\hline & Bachelor's Degree & 81.07 & 82.5 & 85 & 9.34 \\
\hline & Master's Degree & 80.83 & 82.5 & No & 12.30 \\
\hline \multirow[t]{3}{*}{ Living Arrangements } & Extended Family & 75.45 & 82.5 & 65 & 18.67 \\
\hline & Care Centre/ Retirement Village & 56.25 & 58.75 & 52.5 & 13.59 \\
\hline & Living Alone & 86.25 & 90 & No & 13.97 \\
\hline \multirow[t]{2}{*}{ Work Status } & Fully Retired & 68.92 & 66.25 & 65 & 17.51 \\
\hline & Partially Retired & 86.25 & 87.5 & No & 11.15 \\
\hline \multirow[t]{4}{*}{ Health Status } & Ok & 67.5 & 67.5 & No & 2.5 \\
\hline & Could be better & 58.75 & 60 & No & 20.19 \\
\hline & Good & 76.66 & 72.5 & 65 & 17.53 \\
\hline & Excellent & 81.56 & 83.75 & 82.5 & 12.74 \\
\hline
\end{tabular}


The standard deviation is also just 17.75 which shows that most of the scores are close to median. We also encouraged OAs to comment freely on this system for any potential improvements. OAs felt at ease while using the system in the presence of the researcher and gave mostly positive feedback. They said that GoldenJubilants is a source of engagement as it is connecting like-minded people, and it is definitely filling a gap as there is a lack of such kind of systems in Ireland. Below is a sample quotation from a 67-year old female who used our system and answered our questionnaire:

"I think it's really clever. I like the idea of being able to connect with other people, who have the same interest, you know, like reading or walking. I think the news section is really nice as well, because I think it's really nice to keep up with what's going around".

To conclude, the system appeared to be useful for OAs, but a few changes were highlighted according to their preferences. They suggested a change in colour-scheme, increase in font-size, inclusion of relevant photographs that the OA can relate to and an option of volunteering in a group. OAs also approved the need for advanced features such as health reminders, voice recognition, vetting the users of GoldenJubilants and a help section. We intend to incorporate the feedback and new features in the next version of the system and then re-test it to understand if there are any improvements in SUS scores. These proposed additional features include:

- Communication as by-product: The software system should have little focus on virtual communication and more emphasis on active social engagement i.e., a mean to an end or gateway to broker relationships that will end up with face-to-face meetings.

- Accessibility and Formatting: Scrolling should be avoided and input controls should not have a pre-specified range. Colours and pictures must be relevant and attractive. OAs like consistent and large font, buttons and picture size.

- Abstraction: The complex features should remain hidden with only those that are needed made available initially to boost ease of use and familiarity with navigation.

- Overcome privacy and negative perceptions: All of the identifiable details of OAs should be encrypted. Moreover, this can be achieved by sharing stories of those OAs who are already using these systems as testimonials.

\section{DISCUSSION}

The findings of this study are promising, indicating a high level of usability of our proposed system. The features that made GoldenJubilants useful for OAs include the ability to make connections with like-minded people, access to versatile and up-todate news and the management of daily activities in a simple, easy and private way in one location. The augmentation of the SUS with semi-structured questions highlighted potential improvements. These results will help software engineers designing and developing technologies for OAs in the future. As this is a potential growing market, it is important that software engineers understand these requirements and ensure that simplicity and privacy are top priority when designing and developing this type of software systems for OAs.

\subsection{Validity}

We have identified threats to the validity of this survey. From an external validity perspective, we used a convenience sampling approach where participants are from Ireland, have access to the internet, are already familiar with technology and are interested in volunteering. Therefore, our results are not generalizable for the whole OA population. From an internal perspective, the majority of the questions helped us understand the perceived usability of the GoldenJubilants system. However, although we used a standard measure, SUS, some respondents found it difficult to understand some of the questions. In addition, the alternative positive and negative nuanced questions required close attention by the participants and the possibility of error exists. Also, the presence of the researcher during the usability sessions might have biased some of the results.

\section{CONCLUSION AND FUTURE WORK}

This study has demonstrated an overall positive evaluation of GoldenJubilants and revealed that it should be easy to use for OAs in its current state. This level of usability can be further improved by incorporating the feedback which OAs gave during the follow-up interviews. These findings show good potential for the use of SNSs to encourage active social engagement. In addition, we have identified some general requirements which can be used when developing systems for OAs. We intend to revise our system and conduct the usability test again with a larger group of respondents. Following that, a comparison of scores will be made to understand if improvements in the system have been achieved and whether they have any impact on the perceived usability of GoldenJubilants.

\section{ACKNOWLEDGEMENTS}

This work was supported, in part, by Science Foundation Ireland grant no. 13/RC/2094, IBM Damastown Campus Dublin, Ireland \& Ireland Smart Ageing Exchange. We thank all the participants in our study, and Dr. Marcelo Luiz Monteiro Marinho, who facilitated our usability sessions. 


\section{APPENDIX - SUS QUESTIONS (BROOKE, J., 1996)}

1. I think that I would like to use this system frequently.

2. I found the system unnecessarily complex.

3. I thought the system was easy to use.

4. I think that I would need the support of a technical person to be able to use this system.

5. I found the various functions in this system were well integrated.

6. I thought there was too much inconsistency in this system.

7. I would imagine that most people would learn to use this system very quickly.

8. I found the system very cumbersome to use.

9. I felt very confident using the system.

10. I needed to learn a lot of things before I could get going with this system.

\section{REFERENCES}

Ageing Well Network (2012). The New Agenda on Ageing: to make Ireland the best country to grow old in. Dublin.

Ahmad, B., Richardson, I. and Beecham, S. (2017). A Systematic Literature Review of Social Network Systems for Older Adults. International Conference on Product-Focused Software Process Improvement, Innsbruck, Austria, pp. 482-496, Springer, Cham.

Ahmad, B., I. Richardson, S. McLoughlin and Beecham, S. (2018a). Older Adults' Interaction with Mobile Devices in Ireland: A Survey. The 20th International Conference on HumanComputer Interaction with Mobile Devices and Services. September 3rd - 6th, Barcelona, Spain. (in press)

Ahmad, B., I. Richardson, S. McLoughlin and Beecham, S. (2018b). Rewire after you Retire: A customized social volunteering network support system for Older Adults. The 20th International ACM SIGACCESS Conference on Computers and Accessibility, ASSETS, Galway, Ireland. (in press)

Bangor, A., Kortum, P.T. and Miller, J.T. (2008). An empirical evaluation of the system usability scale. International Journal of Human-Computer Interaction, Vol 24(6), pp. 574-594.

Bangor, A., Kortum, P. and Miller, J. (2009). Determining what individual SUS scores mean: Adding an adjective rating scale. Journal of usability studies, Vol 4(3), pp. 114-123.

Brooke J. (1996). SUS- A quick and dirty usability scale, Usability evaluation in industry, Vol 189(194), pp. 4-7.
Burmeister, O.K. (2012). What seniors value about online community. The Journal of Community Informatics, Vol 8(1).

Cornejo, R., Hernandez, D., Favela, J., Tentori, M., Ochoa, S. (2012). Persuading older adults to socialize and exercise through ambient games. 6th International Conference on Pervasive Computing Technologies for Healthcare (PervasiveHealth) and Workshops, pp. 215-218.

Fang, W.C., Hsieh, M.C., Yang, P.C., Li, W.G., Chiu, C.J., Chiang, J.H. (2015). IDianNao: An orange technology that recommends volunteer opportunities to older adults. International Conference on Orange Technologies (ICOT).

Gibson, L., Moncur, W., Forbes, P., Arnott, J., Martin, C. and Bhachu, A.S. (2010). Designing social networking sites for older adults. The 24th BCS Interaction Specialist Group Conference, pp. 186-194.

Lee, Y., Huang, M.C., Zhang, X., Xu, W. (2015). Fridgenet: $A$ nutrition and social activity promotion platform for aging populations. IEEE Intelligent Systems, Vol 30(4), pp. 23-30.

Leist, A.K. (2013). Social media use of older adults: a mini-review. Gerontology, Vol 59(4).

Lewis, J R \& Sauro, J. (2009). The Factor Structure Of The System Usability Scale. The Human Computer Interaction International Conference (HCll 2009), San Diego CA, USA.

Lüders, M. and Brandtzæg, P.B. (2017). 'My children tell me it's so simple': A mixed-methods approach to understand older non-users' perceptions of Social Networking Sites. New media \& society, Vol 19(2), pp.181-198.

Tullis, T., \& Albert, W. (2008). Measuring the user experience: Collecting, analyzing, and presenting usability metrics. Morgan Kaufmann.

Tullis, T., \& Stetson, J. (2004). A comparison of questionnaires for assessing website usability. In Usability Professionals Association (UPA) 2004 Conference, pp. 7-11.

United Nations (2002). World population ageing: 1950-2050. New York: Department of Economic and Social Affairs.

Xie, B., Watkins, I., Golbeck, J. and Huang, M., (2012). Understanding and changing older adults' perceptions and learning of social media. Educational gerontology, Vol 38(4), pp. 282-296. 